\title{
Electrocatalytic Properties of Co-Mo Alloys Electrodeposited from a Citrate-Pyrophosphate Electrolyte
}

\author{
V. S. Kublanovsky • Yu S. Yapontseva
}

Published online: 30 April 2014

(C) The Author(s) 2014. This article is published with open access at Springerlink.com

\begin{abstract}
The electrocatalytic activity of electrodeposits of cobalt and Co-Mo alloys in the electroreduction reaction of hydrogen evolution in an acid, neutral and alkaline media has been studied in this work. To deposit coatings, a nontoxic citrate-pyrophosphate electrolyte was used, which was proposed earlier. It has been found that the alloys having the same chemical composition but a different phase composition can exhibit different electrocatalytic activity. The best electrolyte for the making of catalytically active Co-Mo alloys, for which the decrease in hydrogen evolution overpotential as compared with pure cobalt is about $400 \mathrm{mV}$ at $30 \mathrm{~mA} \mathrm{~cm}^{-2}$ in alkaline solution, is solution with the cobalt and molybdenum concentration ratio 10:1.
\end{abstract}

Keywords Hydrogen evolution · Electrocatalysis . Electrodeposition of Co-Mo alloys

\section{Introduction}

The amount of energy consumed in the world has been steadily increasing in recent decades, and the nonrenewable fossil energy sources account for over $80 \%$ of it [1]. In view of this, the search for efficient, environmentally appropriate methods for the generation of renewable energy is a vital present-day scientific problem [2]. One of such promising trends is fuel cells $[3,4]$, the operation of which is directly bound up with the availability of high-purity hydrogen.

At the present time, the wide use of pure hydrogen is economically inexpedient in comparison, e.g. with the use of natural gas, because of the high cost of its production. The

V. S. Kublanovsky $(\bowtie) \cdot$ Y. S. Yapontseva

V.I. Vernadskii Institute of General and Inorganic Chemistry of the Ukrainian NAS, Prospect Palladina 32-34, Kiev 03142, Ukraine

e-mail: kublan@ukr.net industrial method for hydrogen production by the electrolysis of alkaline aqueous solutions [5] has a number of disadvantages, such as the large expenditure of energy, low efficiency of the process, and the necessity of using noble metals as catalysts. Low-carbon steel, which is corrosion-nonresistant in alkaline medium, and nickel also possess a catalytic effect in the hydrogen evolution reaction [6]; nevertheless, the problem of search for electrode materials with lower hydrogen evolution overpotential and high corrosion resistance remains vital. For instance, platinum group catalysts can be replaced by cheaper materials, such as nickel, molybdenum and ironchromium and iron-manganese alloys, in electrolysis in ionic liquids [7]. However, if the high cost of ionic liquids themselves is taken into account, there is no considerable reduction in the cost of materials for pure hydrogen production.

The main factors in choosing the electrode material are its catalytic activity and corrosion and erosion resistance in the solutions used. It is known from literature that these requirements are met by transition metal alloys, namely, molybdenum and tungsten alloys of iron subgroup metals, which show electrocatalytic properties towards the hydrogen evolution reaction in both acid [8-11] and alkaline aqueous solutions [12-15], and that the use of the electrochemical method for the deposition of thin alloy coatings makes it possible to reduce the cost of electrode materials and hence the cost of produced hydrogen.

On the basis of investigations carried out by the authors of [16], it was concluded that molybdenum-bearing alloys are more active in the hydrogen evolution reaction in alkaline medium than tungsten-bearing alloys. When investigating the properties of Ni-Mo, Ni-W, Co-Mo and Co-W alloys, it was also found that cobalt alloys show a higher catalytic activity than nickel alloys.

On the basis of the data presented in $[12,15]$ for Co-Mo alloys electrodeposited from citrate-ammonium electrolytes, for which electrocatalytic activity in the hydrogen evolution 
reaction in alkaline medium has been proved, we present in this paper investigations of the electrocatalytic properties of similar alloys deposited from a nontoxic citratepyrophosphate electrolyte.

Oxyacid-based (citrate and tartrate) electrolytes are used for the deposition of Co-Mo alloys. The main disadvantage of such electrolytes is that it is impossible to obtain coatings with high molybdenum content since increasing the concentration of molybdate in electrolyte leads to a considerable decrease in the current yield of alloy. A number of such additives as ammonia, ammonium and EDTA allow the coating deposition rate and molybdenum content to be increased, but the current yield decreases with increasing electrolyte $\mathrm{pH}$, and since the cathode layer is alkalized during electrolysis, the electrolyte is unstable during long-duration electrolysis.

Among the electrolytes containing no oxyacids is, for example, pyrophosphate electrolyte, which allows one to obtain alloys containing up to $60 \% \mathrm{Mo}$, but a disadvantage of this electrolyte is the presence of nonmetallic impurities (up to $11 \mathrm{wt} \%$ ), which gives rise to considerable internal stresses in the coating, which results in its cracking and decrease in adhesion to the substrate.

We proposed a citrate-pyrophosphate electrolyte, which combines the advantages of citrate and pyrophosphate electrolytes, with high deposition rate and quality of coatings.

In [12] and [15], a citrate-ammonium electrolyte was used. This electrolyte is a polyligand one, as that developed by us; it allows one to obtain coatings with high molybdenum content and current yield unlike pure citrate electrolyte. In this respect, the main distinction of our work is the nontoxicity of the citrate-pyrophosphate solutions used by us, and a manifest advantage is obtaining of sound and functional coatings without the use of ammonia and ammonium.

\section{Experimental Procedure}

\section{Electrodeposition of Cobalt and Co-Mo Alloys}

Coatings were deposited in a thermostated cell under galvanostatic conditions with the aid of a LIPS-35 dc source. A copper end electrode of $0.5 \mathrm{~cm}$ in diameter was used as the working electrode. $\mathrm{pH}$ was maintained at 8.5. Platinum was used as the anode. All experiments were made under forced convection conditions at a rotary speed of $300 \mathrm{rpm}$ of the magnetic stirrer.

Cobalt and Co-Mo alloys were deposited from a citratepyrophosphate electrolyte, proposed earlier [17], with different concentration ratio of metals in the solution, in a current density range of $10-100 \mathrm{~mA} \mathrm{~cm}{ }^{-2}$ at $50{ }^{\circ} \mathrm{C}$.

The micrographs of the surface of alloys have been obtained on a TESCAN VEGA scanning electron microscope, and the quantitative analysis has been carried out by means of a system for the investigation of chemical composition INCA Energy EDX.

\section{Electrochemical Measurements}

The electroreduction of hydrogen evolution on Co-Mo alloys was studied in the solutions of different mineralization and $\mathrm{pH}$, namely, $0.01 \mathrm{~mol} \mathrm{l}^{-1} \mathrm{H}_{2} \mathrm{SO}_{4}, 0.5 \mathrm{~mol} \mathrm{lL}^{-1} \mathrm{Na}_{2} \mathrm{SO}_{4}$ and $1.0 \mathrm{~mol} \mathrm{lL} \mathrm{L}^{-1} \mathrm{KOH}$.

The voltammetric measurements to determine the kinetic parameters of the hydrogen evolution reaction were made at $25^{\circ} \mathrm{C}$ in a thermostated cell with separate cathode and anode chambers. A platinum wire was used as the auxiliary electrode, and a saturated silver-chloride electrode was used as the reference electrode. All potentials in the paper are given with respect to this reference electrode. To record j-E plots, a PI50-1.1 potentiostat and a PR-8 programmer were used. The current-potential curves were recorded under potentiostatic conditions with a step of $20 \mathrm{mV}$. Before each experiment, argon was passed through the solution during $30 \mathrm{~min}$.

\section{Results and Discussion}

In order to show visually the catalytic effect of alloy in comparison with an individual metal, we have obtained current-potential curves of hydrogen evolution reaction on pure cobalt deposited from a citrate-pyrophosphate electrolyte (electrolyte no. 1, Table 1) 1 at a current density of $30 \mathrm{~mA} \mathrm{~cm}{ }^{-2}$ and a temperature of $50{ }^{\circ} \mathrm{C}$.

Figure 1 shows current-potential curves of electroreduction of hydrogen evolution reaction in an acid, an alkaline and neutral media at cathodes of electrolytic cobalt and Co-Mo alloy with high percentage of molybdenum electrodeposited at the same current density and temperature from an electrolyte with the highest sodium molybdate concentration.

Because of differences in electroreduction mechanism at different $\mathrm{pH}$ values, the lowest hydrogen evolution overpotential at cobalt is observed in acid medium and the highest overpotential in neutral medium, as is the case with the dependence obtained for a mercury electrode and described in

Table 1 Component concentration $\left(\mathrm{mol} \mathrm{l}^{-1}\right)$ in electrolytes for the electrodeposition of Co and Co-Mo alloys

\begin{tabular}{llllll}
\hline Electrolyte no. & $\mathrm{CoSO}_{4}$ & $\mathrm{Na}_{2} \mathrm{MoO}_{4}$ & $\mathrm{Na}_{3} \mathrm{Cit}$ & $\mathrm{K}_{4} \mathrm{P}_{2} \mathrm{O}_{7}$ & $\mathrm{Na}_{2} \mathrm{SO}_{4}$ \\
\hline 1 & 0.1 & 0 & 0.2 & 0.2 & 0.5 \\
2 & 0.1 & 0.005 & 0.2 & 0.2 & 0.5 \\
3 & 0.1 & 0.010 & 0.2 & 0.2 & 0.5 \\
4 & 0.1 & 0.020 & 0.2 & 0.2 & 0.5 \\
5 & 0.1 & 0.100 & 0.2 & 0.2 & 0.5 \\
\hline
\end{tabular}

Cit citrate ions 

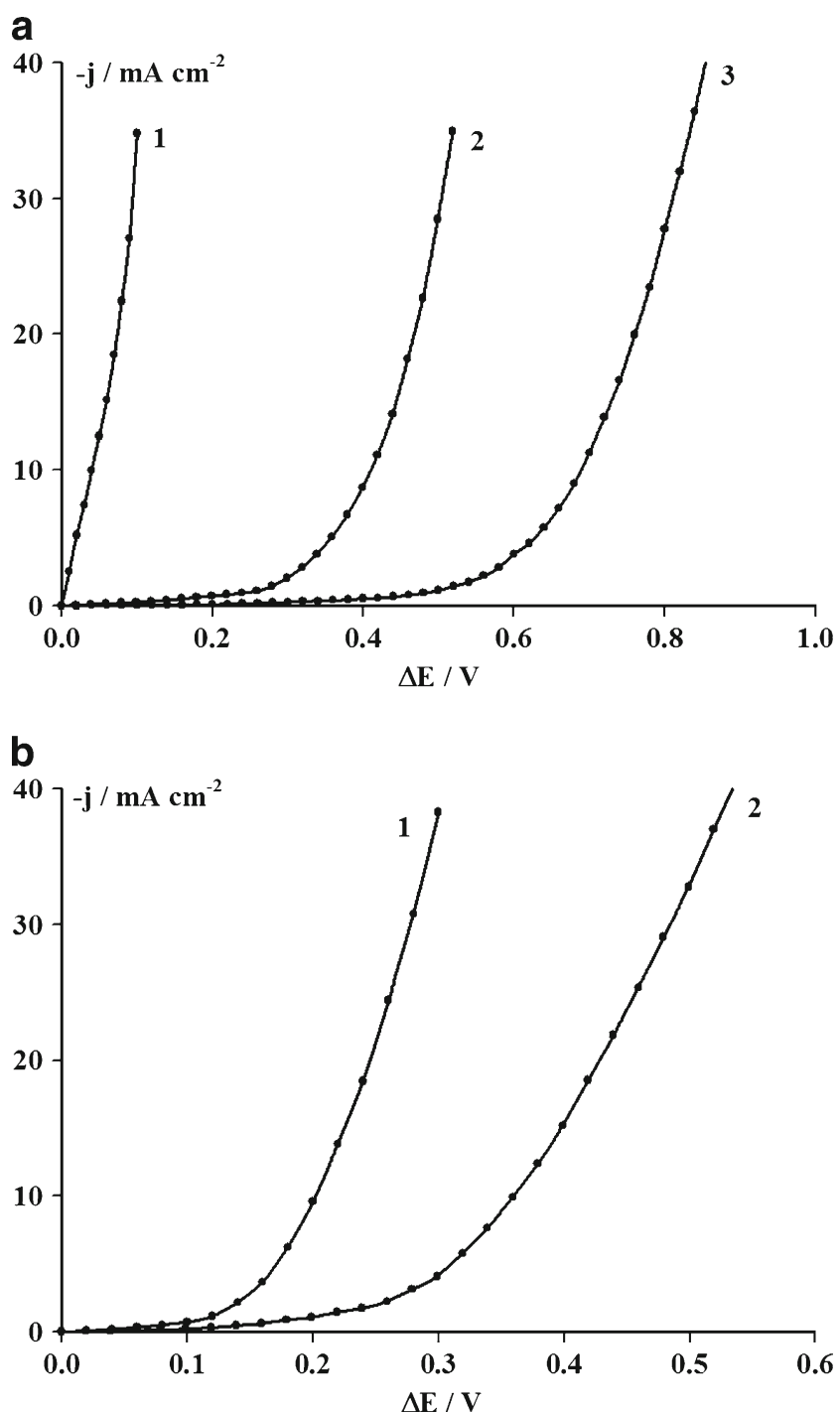

Fig. 1 Stationary current-potential curves of the electroreduction of hydrogen evolution on electrolytic cobalt (a) and Co-Mo alloys (b) with the ratio $[\mathrm{Mo}] /([\mathrm{Mo}]+[\mathrm{Co}])=0.33$ electrodeposited at the current density $30 \mathrm{~mA} \mathrm{~cm}{ }^{-2}$ from the electrolyte no. 5 in media with different $\mathrm{pH}: 1$ $\mathrm{H}_{2} \mathrm{SO}_{4}, 2 \mathrm{KOH}, 3 \mathrm{Na}_{2} \mathrm{SO}_{4}$

[18]. In the case of cobalt cathode, the coefficient $b$ of the Tafel portion of polarization curve for acid, alkaline and neutral media was $0.122,0.142$ and $0.125 \mathrm{~V}$, respectively, and the exchange current density was $1.93,4.59 \times 10^{-2}$ and $6.31 \times 10^{-3} \mathrm{~mA} \mathrm{~cm}^{-2}$.

In Fig. 1, a considerable decrease in hydrogen evolution overpotential at alloy can be seen. For instance, at the current density of $10 \mathrm{~mA} \mathrm{~cm}{ }^{-2}$, the overpotential in alkaline medium decreases by over $200 \mathrm{mV}$.

The plots shown in Fig. 2 have been obtained on alloys electrodeposited at different current densities of 10 $100 \mathrm{~mA} \mathrm{~cm}{ }^{-2}$. The figure presents the ratio of amounts of metals in the alloy and not the exact percentage because of the peculiarities of EDX analysis, in which the coating surface layer, on which a large amount of oxygen and carbon is

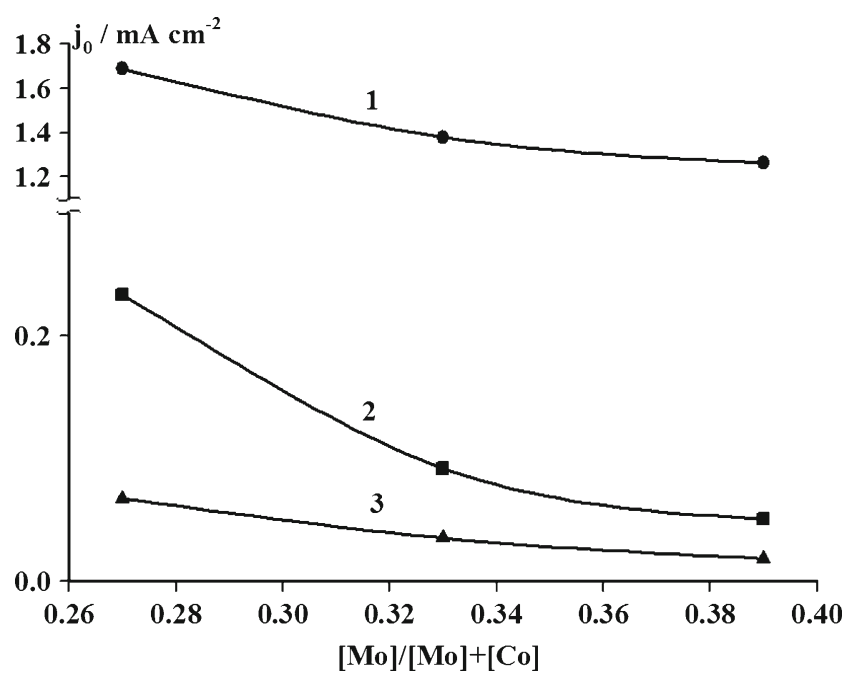

Fig. 2 Dependence of the exchange currents of the electroreduction reaction of hydrogen evolution on the molybdenum/cobalt ratio in alloys electrodeposited at the current densities 10,30 and $100\left(\mathrm{~mA} \mathrm{~cm}^{-2}\right)$ from the solution no. 5 in different media: $1 \mathrm{H}_{2} \mathrm{SO}_{4}, 2 \mathrm{KOH}, 3 \mathrm{Na}_{2} \mathrm{SO}_{4}$

adsorbed, is analyzed. The adsorbed light elements can be removed by polishing the surface or by treating it with argon ions. In both cases, the ratio of the metals in the alloy remains constant over a coating depth of up to $100 \mathrm{~nm}$, as was shown in [15]. Thus, the ratio of molybdenum and cobalt in the surface layer, determined by EDX analysis, corresponds to the volumetric chemical composition of the alloy.

In the alloys deposited from the electrolyte no. 5, the amount of molybdenum decreases, and the exchange current density of the reaction of hydrogen evolution increases on increasing the deposition current density; this may be accounted for by the value which the surface morphology and the true electrode area have, as was shown in [15]. Figure 3 shows photographs of the surface of alloys electrodeposited at two current densities of 10 and $100 \mathrm{mAcm}^{-2}$. From the data presented, it follows that at low current density, smooth, fine crystalline and lustrous coatings are deposited in spite of the presence of microcracks. Increasing the deposition current density to $100 \mathrm{~mA} \mathrm{~cm}^{-2}$ results in the formation of spherulites and considerable surface extension. This regularity is observed for all investigated solutions.

The conclusion that electrocatalytic activity decreases with increasing refractory metal content may contradict papers, published earlier, on the properties of Co-Mo coatings $[12,15]$. However, notice should be taken of how the coating composition was changed. There are several ways of influencing the chemical composition of alloys: changing the composition of the electrolyte, its $\mathrm{pH}$, temperature, current density, hydrodynamic conditions, etc. For instance, in [12], a change in alloy composition is achieved by changing both deposition current density and electrolyte composition, and in [15], by changing $\mathrm{pH}$ and hence the concentration ratio of 
Fig. 3 Morphology of Co-Mo alloys deposited from the electrolytes no. 5 (a, b) and no. 4 (c, d) at the current densities $\left(\mathrm{mA} \mathrm{cm}{ }^{-2}\right) ;(\mathbf{a}, \mathbf{c}) 10,(\mathbf{b}, \mathbf{d}) 100$ and with the ratio $[\mathrm{Mo}] /([\mathrm{Mo}]+$ $[\mathrm{Co}]$ ) equals $0.39,0.27,0.28$ and 0.21 of the metals accordingly
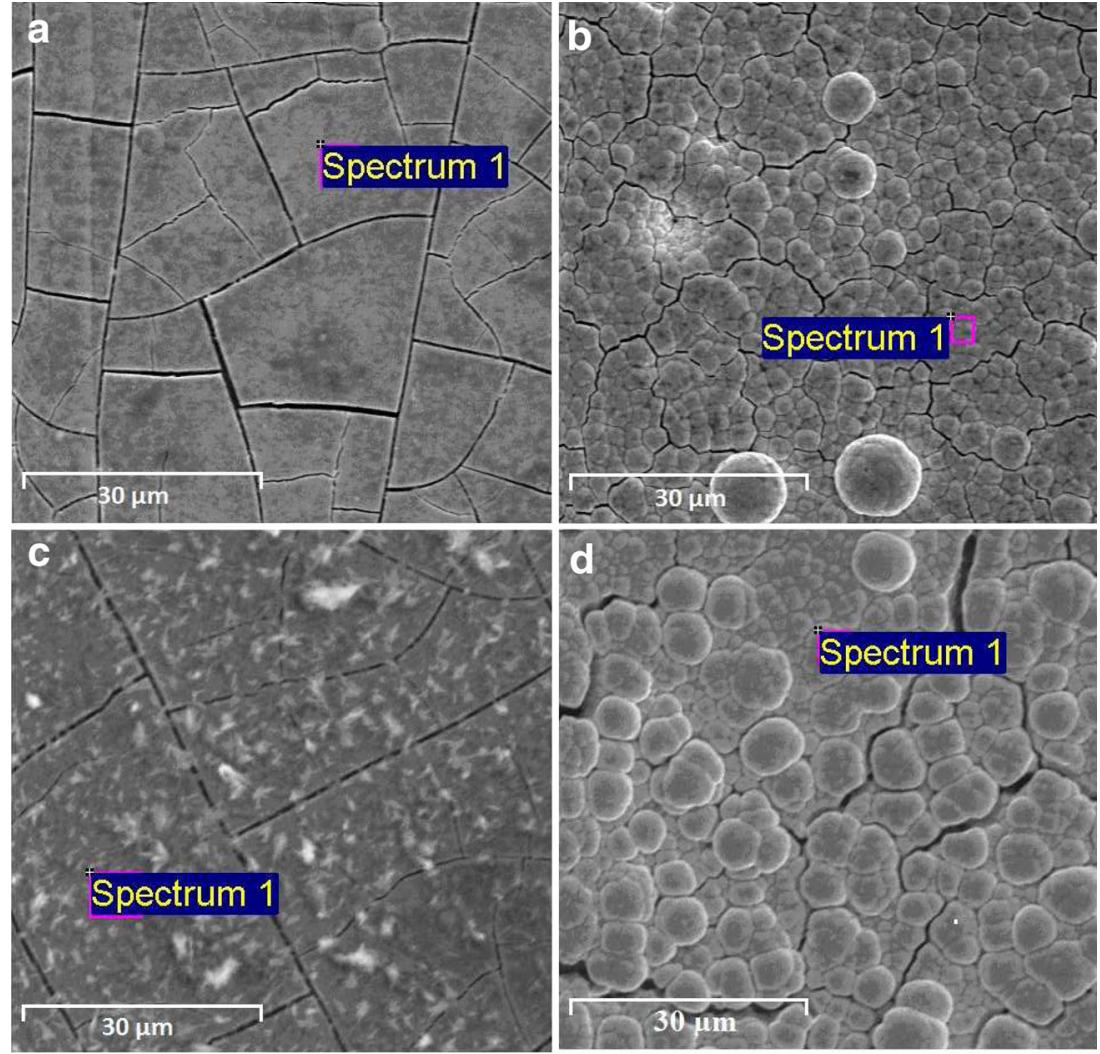

different complex species that are able to be discharged to form an alloy. Thus, it is impossible to obtain a rigorous dependence of catalytic activity on the composition of coatings deposited under different conditions because the electrolysis conditions influence not only chemical but also phase composition, which, in turn, influence the properties of the alloy as a whole.

The effect of the change of the composition of alloy electrodeposition solution on electrocatalytic properties is clearly seen in Fig. 4, which shows current-potential curves of electroreduction of hydrogen evolution on cobalt and alloys obtained at a current density of $30 \mathrm{~mA} \mathrm{~cm}^{-2}$ from electrolytes with different sodium molybdate concentrations. It is seen from the figure that the highest hydrogen evolution overpotential is observed at cobalt deposits and the lowest at the alloy obtained from the electrolyte no. 3 Table 1 . The kinetic parameters of the electroreduction of hydrogen evolution in an alkaline medium are listed in Table 2.

The difference in hydrogen exchange current magnitude on alloys with the same chemical composition can be seen in Fig. 5. In Fig. 5, notice should be taken of how the current efficiency of the alloy in the case of its deposition and its electrocatalytic activity correlate with each other. In spite of the fact that the current yield was measured for a deposition citrate-pyrophosphate electrolyte, this electrolyte is a slightly alkaline salt solution, in which the formed alloys, too, show electrocatalytic activity against concurrent cathodic process, namely, electroreduction of hydrogen evolution; therefore, the

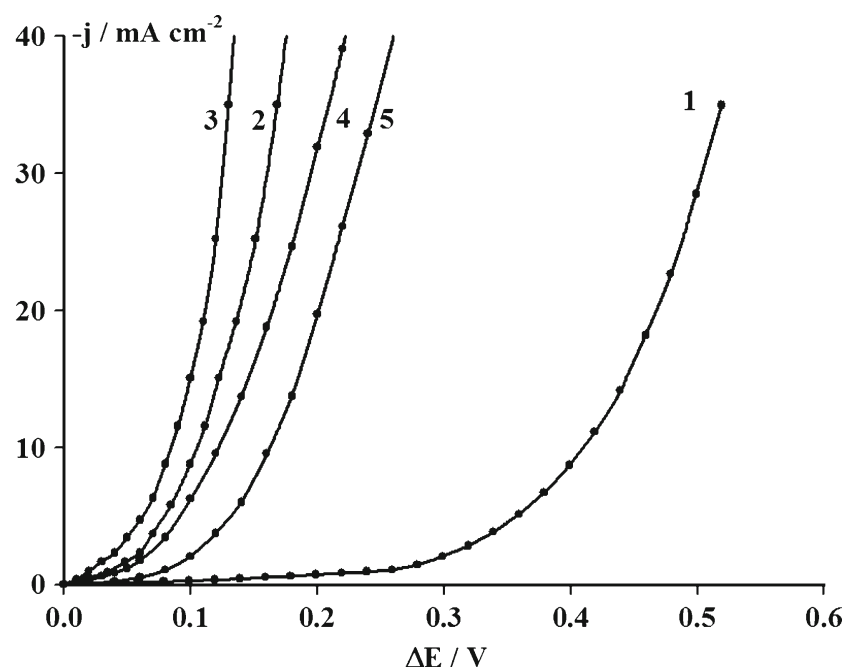

Fig. 4 Polarization curves obtained in a $\mathrm{KOH}$ solution on cobalt and CoMo alloys with the ratio $[\mathrm{Mo}] /([\mathrm{Mo}]+[\mathrm{Co}])$ equals $11,0.19,0.23$ and 0.33 of the metals accordingly electrodeposited at the current density $30 \mathrm{~mA} \mathrm{~cm}^{-2}$ from the electrolytes nos. 1-5 
Table 2 Kinetic parameters of the reduction reaction of hydrogen in a $1 \mathrm{~mol} \mathrm{l}^{-1} \mathrm{KOH}$ solution

\begin{tabular}{|c|c|c|c|c|c|c|}
\hline Electrolyte & $j_{\text {deposition }} / \mathrm{mA} \mathrm{cm}^{-2}$ & $E_{0} / \mathrm{B}$ & $a$ & $b$ & $j_{0} / \mathrm{mA} \cdot \mathrm{cm}^{-2}$ & {$[\mathrm{Mo}] /([\mathrm{Mo}]+[\mathrm{Co}])^{*}$} \\
\hline 1 & 30 & -0.821 & 0.190 & 0.142 & $4.59 \cdot 10^{-2}$ & - \\
\hline \multirow[t]{2}{*}{2} & 10 & -0.910 & 0.181 & 0.1346 & $4.46 \cdot 10^{-2}$ & 0.22 \\
\hline & 30 & -0.985 & 0.038 & 0.077 & $3.21 \cdot 10^{-1}$ & 0.11 \\
\hline \multirow[t]{3}{*}{3} & 10 & -0.940 & 0.125 & 0.091 & $9.23 \cdot 10^{-2}$ & 0.25 \\
\hline & 20 & -1.005 & 0.045 & 0.058 & $1.67 \cdot 10^{-1}$ & 0.23 \\
\hline & 30 & -1.025 & 0.025 & 0.056 & $3.58 \cdot 10^{-1}$ & 0.19 \\
\hline \multirow[t]{3}{*}{4} & 10 & -0.920 & 0.130 & 0.155 & $1.25 \cdot 10^{-1}$ & 0.28 \\
\hline & 30 & -0.980 & 0.050 & 0.062 & $1.56 \cdot 10^{-1}$ & 0.23 \\
\hline & 100 & -1.000 & 0.035 & 0.053 & $2.19 \cdot 10^{-1}$ & 0.21 \\
\hline \multirow[t]{5}{*}{5} & 10 & -0.900 & 0.189 & 0.146 & $5.08 \cdot 10^{-2}$ & 0.39 \\
\hline & 20 & -0.935 & 0.110 & 0.089 & $5.81 \cdot 10^{-2}$ & 0.37 \\
\hline & 30 & -0.980 & 0.083 & 0.080 & $9.17 \cdot 10^{-2}$ & 0.33 \\
\hline & 40 & -0.965 & 0.069 & 0.077 & $1.27 \cdot 10^{-1}$ & 0.30 \\
\hline & 100 & -1.010 & 0.043 & 0.068 & $2.33 \cdot 10^{-1}$ & 0.27 \\
\hline
\end{tabular}

$J_{\text {deposition }}$ is alloy deposition current density, $E_{0}$ is the stationary potential of alloy in the solution, $a$ and $b$ are coefficients in the Tafel equation, $j_{0}$ is hydrogen exchange current density

*Ratio of metals is calculated, from their atomic fraction in alloys

plots shown in Fig. 5 are of antibate character. Thus, though the alloys electrodeposited at high current densities of up to $100 \mathrm{~mA} \mathrm{~cm}{ }^{-2}$ have the highest catalytic activity (for all deposition electrolytes), they deposit with the lowest current yield and with the most developed surface.

It may be concluded from the data obtained that in the case of Co-Mo alloys electrodeposited from citrate-pyrophosphate electrolytes, the hydrogen exchange current decreased for each particular electrolyte on increasing the amount of molybdenum in the alloy. However, in a series of electro-

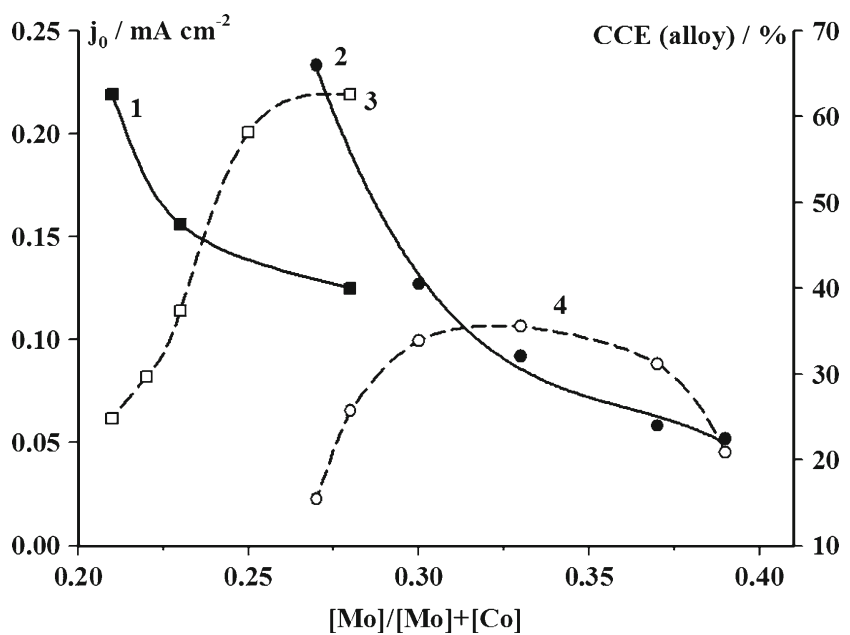

Fig. 5 Dependence of the exchange current of the reduction of hydrogen evolution in a $1 \mathrm{~mol} \mathrm{l}^{-1} \mathrm{KOH}$ solution (curves 1,2) and current yield in the electrodeposition of Co-Mo alloys (curves 3, 4) from the electrolytes no. 4 and no. 5 on the metal ratio in the alloy lytes with different concentration ratio of the metals being discharged, alloys that differ greatly in chemical composition are able to show the same electrocatalytic activity; this can be seen in Fig. 6, which presents values of hydrogen exchange current and stationary potentials for alloys deposited at the same current density but from different electrolytes.

An 'extremal' nature is typical both of the plot of exchange current against alloy component ratio and of the plot of stationary potential of these alloys in $\mathrm{KOH}$ solution against alloy component ratio.

Thus, the electrolyte no. 3 with the cobalt and molybdenum concentration ratio 10:1 Table 1, which stands midway in the series of investigated solutions, is the optimal electrolyte for the fabrication of electrocatalytic Co-Mo alloys; hence, increasing or decreasing the concentration of sodium molybdate and hence molybdenum in the alloy impairs the catalytic properties of coating.

It is known [8] that the electrocatalytic activity of the electrode material can be increased in several ways, e.g. by increasing the area of the true working surface of catalyst, namely, by making catalyst not as a continuous film but as nanofibers [19]. The true surface must be estimated when depositing continuous alloy coatings, as this was done in [15]; however, it should be noted that the factor of surface development is not a determining factor in the ascertainment of the causes of the electrocatalytic activity of materials under investigation. Another important factor is the nature of the component metals of the alloy, which was shown in [16], but 


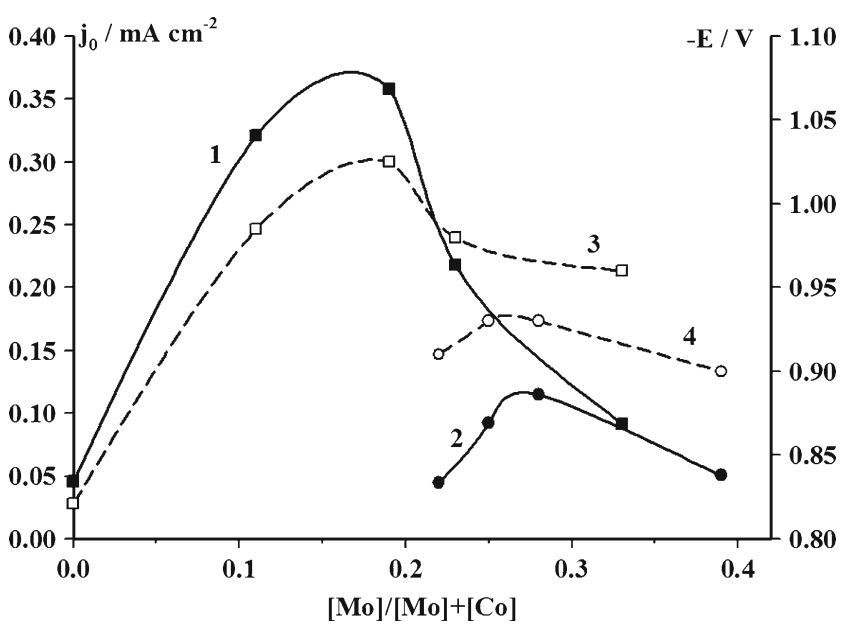

Fig. 6 Dependence of exchange current magnitude (curves 1,2) and stationary potential (curves 3,4 ) in a $\mathrm{KOH}$ solution on the molybdenum/ cobalt ratio in alloys deposited at the current densities $\left(\mathrm{mA} \mathrm{cm}^{-2}\right):(1,3)$ $30,(2,4) 10$

the physicochemical properties of one metal do not account for the synergistic effect in the use of alloys.

The phenomenon that describes most reliably the synergistic action of two and more metals not only in alloys but also even in mechanical mixtures is the so-called spillover effect, which is described, e.g. in [8-10]. This effect manifests itself on the alloys whose components have different properties with respect to hydrogen evolution reaction, namely, a different reaction mechanism is realized on different metals. For instance, on the iron group metals, the step of electron transfer to adsorbed hydrogen ion is fast, and the recombination step is slow, i.e. in our case, cobalt in the alloy will facilitate the accumulation of adsorbed atomic hydrogen on the surface. On the molybdenum surface, the stage of atomic hydrogen removal is fast owing to the electrochemical desorption mechanism.

Due to this, the synergistic effect of alloy will manifest itself when the atoms on the electrocatalyst surface are arranged optimally for the occurrence of the sequential reaction stages: first on cobalt atom and then on molybdenum atom. This is possible when the alloy is not an individual phase of metals and their mechanical mixture, but, e.g. intermetallic compounds or an amorphous phase, or solid solutions with nanosized crystals.

In our paper [20], it was shown that the coatings deposited under different conditions show different corrosion and magnetic characteristics. The study of the phase composition of deposits by X-ray phase analysis did not give any sharp peaks, which indicates either amorphous state of coating or nanometric size of crystals. Therefore, we employed the electrochemical method of linear stripping voltammetric analysis, which showed that the coatings of the same chemical composition have different distribution of elements between the phase of pure cobalt, which is responsible for the presence of magnetic properties, and the CoMo solid solution phase in cobalt, which gives anticorrosion properties to deposits.

\section{Conclusions}

Electrolytic Co-Mo alloys deposited from a citratepyrophosphate electrolyte possess electrocatalytic activity in the hydrogen evolution reaction in comparison with pure cobalt deposited from an analogous complex salt electrolyte. The exchange current of hydrogen reaction is ambiguously bound up with the chemical composition of alloys. For instance, for each electrolyte from the proposed series, the hydrogen exchange current decreases with increasing molybdenum content, but in this case, the alloy deposited from an electrolyte with the cobalt and molybdate concentration ratio 10:1 has the highest activity. That is, increasing or decreasing the amount of molybdenum in the solution and hence in the alloy leads to a decrease in catalyst activity, and alloys of the same chemical composition differ in properties. Probably, in the case of electrolysis in solution with the component ratio 10:1, the optimal conditions are achieved for the formation of amorphous or nanocrystalline coating with the component distribution over the surface which makes spillover effect and hence increase in hydrogen evolution reaction rate possible.

Acknowledgment The work has been carried out within the framework "The Program on Fundamental Studies of the National Academy of Sciences of Ukraine" agreement no. 8-14.

Open Access This article is distributed under the terms of the Creative Commons Attribution License which permits any use, distribution, and reproduction in any medium, provided the original author(s) and the source are credited.

\section{References}

1. P. Moriarty, D. Honner, Hydrogen's role in an uncertain energy future. Int J Hydrogen Energy 34, 31-39 (2009)

2. G. Marba'n, T. Valde's-Solı's, Towards the hydrogen economy. Int J Hydrogen Energy 32, 1625-1637 (2007)

3. M.Z. Jacobson, W.G. Colella, D.M. Golden, Cleaning the air and improving health with hydrogen fuel-cell vehicles. Science $\mathbf{3 0 8}$, 1901-1905 (2005)

4. G. Brumfiel, Hydrogen cars fuel debate on basic research. Nature 422, 104 (2003)

5. O. Ulleberg, Modeling of advanced alkaline electrolyzers: a system simulation approach. Int J Hydrogen Energy 28, 21-33 (2003)

6. J. Divisek, H. Schmitz, B. Steffen, Electrocatalyst materials for hydrogen evolution. Electrochim Acta 39, 1723-1731 (1994)

7. G. Loget, J.C. Padilha, E.A. Martini, M.O. de Souza, R.F. de Souza, Efficiency and stability of transition metal electrocatalysts for the 
hydrogen evolution reaction using ionic liquids as electrolytes. Int $\mathrm{J}$ Hydrogen Energy 34, 84-90 (2009)

8. E. Navarro-Flores, Z. Chong, S. Omanovic, Characterization of Ni, $\mathrm{NiMo}$, NiW and $\mathrm{NiFe}$ electroactive coatings as electrocatalysts for hydrogen evolution in an acidic medium. J Mol Catal A Chem 226, 179-197 (2005)

9. J.G. Highfield, E. Claude, K. Oguro, Electrocatalytic synergism in $\mathrm{Ni} / \mathrm{Mo}$ cathodes for hydrogen evolution in acid medium: a new model. Electrochim Acta 44, 2805-2814 (1999)

10. S. Martinez, M. Metikoš-Huković, L. Valek, Electrocatalytic properties of electrodeposited Ni-15Mo cathodes for the HER in acid solutions: Synergistic electronic effect. J Mol Catal A Chem 245, 114-121 (2006)

11. M. Ved, N. Sakhnenko, T. Bairachnaya, N. Tkachenko, Structure and properties of electrolytic cobalt-tungsten alloy coatings. Functional Materials 15, 613-617 (2008)

12. A. Subramania, A.R. Sathiya Priya, V.S. Muralidharan, Electrocatalytic cobalt-molybdenum alloy deposits. Int J Hydrogen Energy 32, 2843-2847 (2007)

13. R.I. Arul, On the catalytic activity of Ni-Mo-Fe composite surface coatings for the hydrogen cathodes in the industrial electrochemical production of hydrogen. Appl Surf Sci 59, 245-252 (1992)

14. N.V. Krstajic, V.D. Jovic, L.J. Gajic-Krstajic, B.M. Jovic, A.L. Antozzi, G.N. Martelli, Electrodeposition of Ni-Mo alloy coatings and their characterization as cathodes for hydrogen evolution in sodium hydroxide solution. Int J Hydrogen Energy 33, 3676-3687 (2008)

15. V.V. Kuznetsov, A.A. Kalinkina, T.V. Pshenichkina, V.V. Balabaev, Electrocatalytic Properties of Cobalt-Molybdenum Alloy Deposits in the Hydrogen Evolution Reaction. Russ J Electrochem 44, 13501358 (2008)

16. C. Fan, D.L. Piron, A. Sleb, P. Paradis, Study of electrodeposited nickel-molybdenum, nickel-tungsten, cobalt-molybdenum, and cobalt-tungsten as hydrogen electrodes in alkaline water electrolysis. J Electrochem Soc 141, 382-387 (1994)

17. Y.S. Yapontseva, V.A. Gromova, V.S. Kublanovsky, A.I. Dikusar, Electrodeposition of Co-Mo alloys from a citrate-pyrophosphate electrolyte. Ukr. Khim. Zhurn. 74, 44-48 (2008) (in Russian)

18. Antropov L. I., Theoretical Electrochemistry (University Press of the Pacific, 2001), p. 572

19. J.K. Lee, Y. Yi, H.J. Lee, S. Uhm, J. Lee, Electrocatalytic activity of $\mathrm{Ni}$ nanowires prepared by galvanic electrodeposition for hydrogen evolution reaction. Catal Today 146, 188191 (2009)

20. V.A. Gromova, Y.S. Yapontseva, V.S. Kublanovsky, Stripping analysis of electrolytic Co-Mo alloys deposited from a polyligand citratepyrophosphate electrolyte. Metallofiz. Noveishie Tekhnol. 30, 467476 (2008) (in Russian) 